INPLASY

PROTOCOL

To cite: Cao et al. The effect of acupuncture on Postpartum stress urinary incontinence: A protocol for systemic review and meta-analysis. Inplasy protocol 202220045. doi: 10.37766/inplasy2022.2.0045

Received: 13 February 2022

Published: 13 February 2022

Corresponding author:

Fengye Cao

932148446@qq.com

Author Affiliation:

Cao Fengye

Support: 2017-XK-A18.

Review Stage at time of this submission: Preliminary searches.

Conflicts of interest:

None declared.

\section{The effect of acupuncture on Postpartum stress urinary incontinence: A protocol for systemic review and meta-analysis}

Cao, FY1; Zhuansun, QS2; Gan, L³; Lin, XM4.

Review question / Objective: To comprehensively explore the effectiveness and safety of acupuncture in the treatment of Postpartum stress urinary incontinence.

Condition being studied: Postpartum stress urinary incontinence (PSUI) is a phenomenon of urinary involuntary overflow when abdominal pressure suddenly rises.About $\mathbf{4 0} \%$ of postpartum women suffer from urinary incontinence, $30 \%$ of them still suffer from urinary incontinence 3 months after delivery. The both of physical and mental health of the postpartum women can be affected significantly. At present, there is no specific treatment for postpartum SUI, and the surgical effect is good, but the trauma is large and the patient ' $s$ acceptance is low. Therefore, the clinical practice is more inclined to use conservative therapy based on pelvic floor muscle training, but the compliance of pelvic floor muscle training is poor, and the treatment cycle is long. Robust evidences demonstrated that the effectiveness of acupuncture in treating SUI. However, the effectiveness of acupuncture for PSUI is still remain largely uncertain and the relevant evidence is deficiency. Accordingly, the first systematic review and meta-analysis will be conducted to investigate the efficacy and safety of acupuncture in the treatment of PSUI.

INPLASY registration number: This protocol was registered with the International Platform of Registered Systematic Review and Meta-Analysis Protocols (INPLASY) on 13 February 2022 and was last updated on 13 February 2022 (registration number INPLASY202220045).

\section{INTRODUCTION}

Review question / Objective: To comprehensively explore the effectiveness and safety of acupuncture in the treatment of Postpartum stress urinary incontinence.

Condition being studied: Postpartum stress urinary incontinence (PSUI) is a 
phenomenon of urinary involuntary overflow when abdominal pressure suddenly rises.About $40 \%$ of postpartum women suffer from urinary incontinence, $30 \%$ of them still suffer from urinary incontinence 3 months after delivery. The both of physical and mental health of thepostpartum women can be affected significantly. At present, there is no specific treatment for postpartum SUI, and the surgical effect is good, but the trauma is large and the patient ' $s$ acceptance is low. Therefore, the clinical practice is more inclined to use conservative therapy based on pelvic floor muscle training, but the compliance of pelvic floor muscle training is poor, and the treatment cycle is long. Robust evidences demonstrated that the effectiveness of acupuncture in treating SUI. However, the effectiveness of acupuncture for PSUI is still remain largely uncertain and the relevant evidence is deficiency. Accordingly, the first systematic review and meta-analysis will be conducted to investigate the efficacy and safety of acupuncture in the treatment of PSUI.

\section{METHODS}

Participant or population: Patients diagnosed with Postpartum stress urinary incontinence will be included.

Intervention: The interventions under consideration must involve needle insertion at acupuncture points, pain points or trigger points, and had to be described as acupuncture. Studies evaluating the following treatments, including body acupuncture (MA or EA), auricular acupuncture, scalp acupuncture, warm needle acupuncture, plum blossom needling and fire needling, will be considered.

Comparator: The inclusion of the comparator mainly included sham or placebo acupuncture intervention such as non-penetrating, shamneedleor superficial needling at non-acupuncture points, moxibustion, massage, western medicine, pelvic floor muscle training and biofeedback electrical stimulation will also be taken into account.
Study designs to be included: Only randomized controlled trials (RCTs) comparing acupuncture to sham or placebo acupuncture, no treatment, conventional therapies or Chinese herbal medicine for patients with PSUI will be involved.

Eligibility criteria: Inclusion criteria: (1) RCTs involving acupuncture against another treatment or sham in patients with PSUI; or studies that in the term of 'randomization' was mentioned. (2) Participants must be diagnosed with PSUI. No restrictions on age, nationally, and race. (3) The intervention was acupuncture, electro-acupuncture or warm acupuncture and was compared with the control group. Exclusion criteria: (1) Incorrect randomization methods and other designs. (2) Studies comparing between different types of acupuncture therapies, such as only compared different manipulation forms or acupoint-selection methods of acupuncture, will be excluded. (3) Duplicate literature and incomplete data will not be considered.

Information sources: The 8 databases including the PubMed, Web of Science (WoS), EMBASE, the Cochrane Central Register of Controlled Trials (CENTRAL), Chinese National Knowledge Infrastructure (CNKI), Chinese Biomedical Literature Database (CBM), Wanfang Database and Technology Periodical Database (VIP) will be searched by two researchers independently.

Main outcome(s): The pelvic floor muscle strength.

Quality assessment / Risk of bias analysis: Two authors will assess the risk of bias independently by using Cochrane Collaboration's tool for the all included studies. We will evaluate the risk of bias in the following domains: sequence generation, allocation sequence concealment, blinding of participants and personnel and outcome assessors, incomplete outcome data, selective outcome reporting and other sources of bias. In addition, we will grade the quality 
of the evidence based on the Grades Profiler as the Grading of Recommendation, Assessment, Development, and Evaluation (GRADE) system. The assessments will be classified into three levels: low risk, high risk and unclear risk.

Strategy of data synthesis: We will use the Review Manager software V.5.3 to carry out statistical analysis. Mean difference (MD) or standardized means difference (SMD) will be used for continuous data. Risk ratio (RR) or risk difference (RD) will be used for the analysis of dichotomous data. If the $I^{2}$ test is less than $\mathbf{5 0} \%$, the fixed effect model will be used for data synthesis. If the $I^{2}$ test is between $50 \%$ to $75 \%$, the random-effects model will be conducted for data synthesis. And if the $I^{2}$ test is higher than $75 \%$, we will find the possible reasons from both clinical and methodological perspectives and provide an explanation or conduct subgroup analysis.

Subgroup analysis: Subgroup analysis will be executed if data is available. To detect possible heterogeneity of the results, subgroups analysis will be performed about the following four aspects: (1) Chinese studies vs other countries studies; (2) Acupuncture vs controls or different type of sham acupuncture; (3) Length of treatment differences. In addition, subgroup analysis will also be conducted if any significant covariates contributing to the heterogeneity.

Sensitivity analysis: To test the robustness of the review conclusions, a sensitivity analysis will be performed for the primary outcomes. The results of the sensitivity analysis will be presented in summary tables. The risk of bias in the review process as indicated by the results of the sensitivity analysis will be discussed.

Country(ies) involved: China.

Keywords: Postpartum, Stress urinary incontinence, Acupuncture, Meta-analysis.

Contributions of each author:

Author 1 - Fengye Cao.
Author 2 - Qinshuai Zhuansun. Author 3 - Lin Gan.

Author 4 - Xianming Lin. 